\title{
Top 100 cited articles on osteoarthritis from 1990 to 2020
}

Feng Yin ${ }^{1, \#}$, Qiuyu Yang ${ }^{1, \#}$, Yue He ${ }^{2}$, Lihui Peng ${ }^{1}$, Zixia Zhao ${ }^{1}$, Chengsong He ${ }^{1}$, Jie Chen ${ }^{1, *}$

${ }^{1}$ Department of Rheumatology and Immunology, The Affiliated Hospital of Southwest Medical University, Luzhou,

Sichuan Province, China

${ }^{2}$ Department of Ophthalmology, The Affiliated Hospital of Southwest Medical University, Luzhou, Sichuan Province, China

\begin{abstract}
Keywords (OA) from 1990 to 2020. and the number of citations. guideline $(n=12)$, basic science $(n=10)$ and other types.

arthritis • bibliometrics $\bullet$ osteoarthritis $\bullet$ Top-cited

\section{Background}

Osteoarthritis $(\mathrm{OA})$ is an age-associated disease characterized by chronic joint pain resulting from degradation of articular cartilage, inflammation of the synovial lining, and changes to the subchondral bone. ${ }^{[1]} \mathrm{OA}$ is the osteoarticular disease with the highest prevalence worldwide. ${ }^{[2]}$ In addition to being a major source of disability, OA reduced work productivity and lost physical fitness at the individual level, and increased health and welfare costs at the societal level. ${ }^{[3,4]}$
\end{abstract}

Objectives: The purpose of this study was to identify and analyze the 100 top-cited articles in the field of osteoarthritis

Methods: We used the Web of Science to retrieve the articles related to OA. Then we selected 100 target articles and manually collected their general information, including article title, author, year of publication, journal, type of article,

Results: The 100 top-cited articles were published in the period from 1990 to 2015 . These articles have been cited 66,494 times in total, with the highest being 2382 times, the lowest being 433 times, the median number being 613 , and a mean of 664.94 times. The 100 top-cited articles appeared in a total of 35 influential journals. The greatest number of articles in the top of 100 was published in Arthritis and Rheumatism. The authors of these articles came from 18 countries, led by the United States $(n=48)$, followed by the United Kingdom $(n=15)$. Among all the institutions, Boston University led the list with 10 articles. The most prevalent type of the study was review $(n=38)$ and clinical study $(n=38)$, followed by

Conclusions: This study provided some insights on the literature development and citation of OA in the recent 30 years. Articles published in high-impact journals are more likely to be cited in the field of OA. As recent studies did not have enough time to accumulate the number of citations, the latest articles may not be included in the top 100 cited articles.

Bibliometrics is a quantitative tool for the analysis of literature published in a scientific field. ${ }^{[5]}$ Bibliometrics can be defined as the combination of statistics and philology, which uses statistical methods to study the relationship between literature and information. ${ }^{[6]}$ In the last 30 years, many articles have been published on OA. At present, to the best of our knowledge, there were several analyses of the 100 topcited articles in various specialties, such as urological surgery, ${ }^{[7]}$ endodontics, ${ }^{\left[{ }^{[8]}\right.}$ gastric disease..$^{[0]}$ sleep medicine, ${ }^{[10]}$ and robotic surgery. ${ }^{[11]}$ However, no comprehensive study of the top-cited articles in the field of OA has appeared to date. The purpose of this study was to identify and analyze the 100 articles on OA. And the expectation was that this study could provide a reference for the development of OA in the future.

\footnotetext{
Address for correspondence:

"Jie Chen, Department of Rheumatology and Immunology, The Affiliated

Hospital of Southwest Medical University, No. 25, Taiping Street, Jiangyang

District, Luzhou 646000, Sichuan Province 646000, China.

E-mail: cjlion@qq.com (Jie Chen)

\#Feng Yin and Qiuyu Yang contributed equally to this work and should be

considered as co-first authors.
} 


\section{Materials and Methods}

We started searching for information on July 1, 2020. The whole information about the citations was collected from July 1, 2020 to July 15, 2020. The Web of Science was used by us to retrieve articles with "OA" as the subject, and the period was from January 1, 1990 to July 1, 2020. Only articles that focused on the subject of OA and were edited in English were included. The retrieved articles were ranked in descending order according to the number of citations. We selected the top 100 related articles and manually collected their basic information, including titles, the number of citations, authors, journals, and year of publication. Two reviewers (Feng Yin and Qiuyu Yang) independently selected the top 100 cited articles about OA. When there were disagreements between two reviewers, a third reviewer (Yue He) joined and helped achieve consensus. After identifying the 100 target articles, we viewed

Table 1: Top 100 cited articles in the field of $O A$

\begin{tabular}{|c|c|c|}
\hline Rank & Article & No. of citations \\
\hline 1 & Gastrointestinal toxicity with celecoxib vs nonsteroidal anti-inflammatory drugs for osteoarthritis and rheumatoid arthritis: the CLASS study: A RCT. & 2382 \\
\hline 2 & Femoroacetabular impingement: a cause for osteoarthritis of the hip. & 1781 \\
\hline 3 & Knee Injury and Osteoarthritis Outcome Score (KOOS) - development of a self-administered outcome measure. & 1689 \\
\hline 4 & OARSI recommendations for the management of hip and knee osteoarthritis, Part II: OARSI evidence-based, expert consensus guidelines. & 1655 \\
\hline 5 & $\begin{array}{l}\text { American College of Rheumatology } 2012 \text { recommendations for the use of nonpharmacologic and pharmacologic therapies in osteoarthritis } \\
\text { of the hand, hip, and knee. }\end{array}$ & 1566 \\
\hline 6 & Osteoarthritis: new insights. Part 1: the disease and its risk factors. & 1491 \\
\hline 7 & $\begin{array}{l}\text { Recommendations for the medical management of osteoarthritis of the hip and knee: } 2000 \text { update. American College of Rheumatology } \\
\text { Subcommittee on Osteoarthritis Guidelines. }\end{array}$ & 1452 \\
\hline 8 & OARSI guidelines for the non-surgical management of knee, hip, and polyarticular osteoarthritis. & 1346 \\
\hline 9 & $\begin{array}{l}\text { EULAR Recommendations 2003: an evidence-based approach to the management of knee osteoarthritis: Report of a Task Force of the } \\
\text { Standing Committee for International Clinical Studies Including Therapeutic Trials (ESCISIT). }\end{array}$ & 1314 \\
\hline 10 & The American College of Rheumatology criteria for the classification and reporting of osteoarthritis of the hip. & 1310 \\
\hline 11 & A controlled trial of arthroscopic surgery for osteoarthritis of the knee. & 1206 \\
\hline 12 & The long-term consequence of anterior cruciate ligament and meniscus injuries: osteoarthritis. & 1163 \\
\hline 13 & Role of proinflammatory cytokines in the pathophysiology of osteoarthritis. & 1071 \\
\hline 14 & The global burden of hip and knee osteoarthritis: estimates from the global burden of disease 2010 study. & 1061 \\
\hline 15 & Osteoarthritis cartilage histopathology: grading and staging. & 1024 \\
\hline 16 & Osteoarthritis: an update with relevance for clinical practice. & 982 \\
\hline 17 & $\begin{array}{l}\text { OARSI recommendations for the management of hip and knee osteoarthritis: part III: Changes in evidence following systematic cumulative } \\
\text { update of research published through January } 2009 .\end{array}$ & 977 \\
\hline 18 & A randomized trial comparing aerobic exercise and resistance exercise with a health education program in older adults with knee osteoarthritis. & 946 \\
\hline 19 & Osteoarthritis: a disease of the joint as an organ. & 925 \\
\hline 20 & An update on the epidemiology of knee and hip osteoarthritis with a view to prevention. & 874 \\
\hline 21 & Osteoarthritis. & 855 \\
\hline 22 & Long-term effects of glucosamine sulfate on osteoarthritis progression: a randomized, placebo-controlled clinical trial. & 854 \\
\hline 23 & The role of knee alignment in disease progression and functional decline in knee osteoarthritis. & 850 \\
\hline 24 & WORMS of the knee in osteoarthritis. & 847 \\
\hline 25 & Dynamic load at baseline can predict radiographic disease progression in medial compartment knee osteoarthritis. & 845 \\
\hline 26 & High prevalence of knee osteoarthritis, pain, and functional limitations in female soccer players twelve years after an anterior cruciate ligament injury. & 840 \\
\hline 27 & Deletion of active ADAMTS5 prevents cartilage degradation in a murine model of osteoarthritis. & 837 \\
\hline 28 & The KOOS: from joint injury to osteoarthritis. & 834 \\
\hline 29 & Enhanced cleavage of type II collagen by collagenases in osteoarthritic articular cartilage. & 798 \\
\hline 30 & Osteoarthritis, an inflammatory disease: potential implication for the selection of new therapeutic targets. & 790 \\
\hline 31 & Knee pain and osteoarthritis in older adults: a review of community burden and current use of primary health care. & 790 \\
\hline 32 & The American College of Rheumatology criteria for the classification and reporting of osteoarthritis of the hand. & 790 \\
\hline 33 & Glucosamine, chondroitin sulfate, and the two in combination for painful knee osteoarthritis. & 783 \\
\hline 34 & Cloning, expression, and type II collagenolytic activity of matrix metalloproteinase-13 from human osteoarthritic cartilage. & 766 \\
\hline 35 & The role of cytokines in osteoarthritis pathophysiology. & 764 \\
\hline 36 & Stem cell therapy in a caprine model of osteoarthritis. & 760 \\
\hline 37 & Articular cartilage: degeneration and osteoarthritis, repair, regeneration, and transplantation. & 705 \\
\hline
\end{tabular}




\begin{tabular}{|c|c|c|}
\hline Rank & Article & No. of citations \\
\hline 38 & Human autologous culture expanded bone marrow mesenchymal cell transplantation for repair of cartilage defects in osteoarthritic knees. & 704 \\
\hline 39 & Acetabular and femoral anteversion: relationship with osteoarthritis of the hip. & 692 \\
\hline 40 & Atlas of individual radiographic features in osteoarthritis. & 676 \\
\hline 41 & Epidemiology of osteoarthritis. & 658 \\
\hline 42 & Guidelines for the medical management of osteoarthritis. Part II. Osteoarthritis of the knee. American College of Rheumatology. & 655 \\
\hline 43 & The etiology of osteoarthritis of the hip: an integrated mechanical concept. & 655 \\
\hline 44 & Exercise and dietary weight loss in overweight and obese older adults with knee osteoarthritis: the Arthritis, Diet, and Activity Promotion Trial. & 652 \\
\hline 45 & Osteoarthritis as an inflammatory disease (osteoarthritis is not osteoarthrosis!). & 642 \\
\hline 46 & The association of bone marrow lesions with pain in knee osteoarthritis. & 633 \\
\hline 47 & Quadriceps weakness and osteoarthritis of the knee. & 632 \\
\hline 48 & Pathogenesis and management of pain in osteoarthritis. & 625 \\
\hline 49 & Weight loss reduces the risk for symptomatic knee osteoarthritis in women. The Framingham Study. & 618 \\
\hline 50 & Risk factors for the onset of osteoarthritis of the knee in older adults: a systematic review and meta-analysis. & 617 \\
\hline 51 & Atlas of individual radiographic features in osteoarthritis, revised. & 609 \\
\hline 52 & Osteoarthritis: epidemiology. & 609 \\
\hline 53 & Osteoarthritis. & 608 \\
\hline 54 & A framework for the in vivo pathomechanics of osteoarthritis at the knee. & 593 \\
\hline 55 & Glucosamine and chondroitin for treatment of osteoarthritis: a systematic quality assessment and meta-analysis. & 589 \\
\hline 56 & $\begin{array}{l}\text { EULAR evidence-based recommendations for the management of hip osteoarthritis: report of a task force of the EULAR Standing Committee } \\
\text { for International Clinical Studies Including Therapeutics (ESCISIT). }\end{array}$ & 577 \\
\hline 57 & $\begin{array}{l}\text { Recommendations for a core set of outcome measures for future phase III clinical trials in knee, hip, and hand osteoarthritis. Consensus } \\
\text { development at OMERACT III. }\end{array}$ & 571 \\
\hline 58 & Articular cartilage and changes in arthritis. An introduction: cell biology of osteoarthritis. & 565 \\
\hline 59 & Inflammation in osteoarthritis. & 562 \\
\hline 60 & Synovial tissue inflammation in early and late osteoarthritis. & 560 \\
\hline 61 & Osteoarthritis chondrocytes die by apoptosis. A possible pathway for osteoarthritis pathology. & 555 \\
\hline 62 & $\begin{array}{l}\text { Matrix metalloproteinase and proinflammatory cytokine production by chondrocytes of human osteoarthritic cartilage: associations } \\
\text { with degenerative changes. }\end{array}$ & 548 \\
\hline 63 & The OARSI histopathology initiative - recommendations for histological assessments of osteoarthritis in the mouse. & 544 \\
\hline 64 & Metal-on-metal resurfacing of the hip in patients under the age of 55 years with osteoarthritis. & 533 \\
\hline 65 & $\begin{array}{l}\text { Evaluation of clinically relevant changes in patient-reported outcomes in knee and hip osteoarthritis: the minimal clinically important } \\
\text { improvement. }\end{array}$ & 533 \\
\hline 66 & The role of synovitis in pathophysiology and clinical symptoms of osteoarthritis. & 529 \\
\hline 67 & Risk factors for incident radiographic knee osteoarthritis in the elderly: the Framingham Study. & 523 \\
\hline 68 & Sensitization in patients with painful knee osteoarthritis. & 523 \\
\hline 69 & The surgical DMM model of osteoarthritis in the 129/SvEv mouse. & 522 \\
\hline 70 & Incidence of symptomatic hand, hip, and knee osteoarthritis among patients in a health maintenance organization. & 519 \\
\hline 71 & Increased damage to type II collagen in osteoarthritic articular cartilage detected by a new immunoassay. & 516 \\
\hline 72 & The role of the chondrocyte in osteoarthritis. & 513 \\
\hline 73 & EULAR recommendations for the non-pharmacological core management of hip and knee osteoarthritis. & 512 \\
\hline 74 & Bone marrow edema and its relation to progression of knee osteoarthritis. & 511 \\
\hline 75 & Lifetime risk of symptomatic knee osteoarthritis. & 503 \\
\hline 76 & $\begin{array}{l}\text { Prevalence of knee osteoarthritis in the United States: arthritis data from the Third National Health and Nutrition Examination Survey } \\
\text { 1991-94. }\end{array}$ & 499 \\
\hline 77 & Viscosupplementation: a new concept in the treatment of osteoarthritis. & 498 \\
\hline 78 & $\begin{array}{l}\text { High prevalence of osteoarthritis } 14 \text { years after an anterior cruciate ligament tear in male soccer players: a study of radiographic } \\
\text { and patient-relevant outcomes. }\end{array}$ & 491 \\
\hline 79 & Glucosamine sulfate use and delay of progression of knee osteoarthritis: a 3-year, randomized, placebo-controlled, double-blind study. & 489 \\
\hline 80 & $\begin{array}{l}\text { OARSI recommendations for the management of hip and knee osteoarthritis, part I: critical appraisal of existing treatment guidelines } \\
\text { and systematic review of current research evidence. }\end{array}$ & 487 \\
\hline 81 & OMERACT-OARSI initiative: Osteoarthritis Research Society International set of responder criteria for osteoarthritis clinical trials revisited. & 484 \\
\hline 82 & The incidence and natural history of knee osteoarthritis in the elderly. The Framingham Osteoarthritis Study. & 481 \\
\hline 83 & Knee adduction moment, serum hyaluronan level, and disease severity in medial tibiofemoral osteoarthritis. & 480 \\
\hline 84 & A meta-analysis of sex differences prevalence, incidence, and severity of osteoarthritis. & 476 \\
\hline 85 & Risk factors for the incidence and progression of radiographic knee osteoarthritis. & 476 \\
\hline
\end{tabular}




\begin{tabular}{|c|c|c|}
\hline Rank & Article & No. of citations \\
\hline 86 & The WOMAC: a review of its utility and measurement properties. & 467 \\
\hline 87 & Morphologic study of the glenoid in primary glenohumeral osteoarthritis. & 466 \\
\hline 88 & Knee osteoarthritis after meniscectomy: prevalence of radiographic changes after twenty-one years, compared with matched controls. & 459 \\
\hline 89 & Genetic influences on osteoarthritis in women: a twin study. & 458 \\
\hline 90 & The role of synovitis in osteoarthritis pathogenesis. & 456 \\
\hline 91 & Knee osteoarthritis after anterior cruciate ligament injury: a systematic review. & 453 \\
\hline 92 & $\begin{array}{l}\text { Comparison of an antiinflammatory dose of ibuprofen, an analgesic dose of ibuprofen, and acetaminophen in the treatment of patients } \\
\text { with osteoarthritis of the knee. }\end{array}$ & 452 \\
\hline 93 & $\begin{array}{l}\text { Design and conduct of clinical trials in patients with osteoarthritis: recommendations from a task force of the Osteoarthritis Research Society. } \\
\text { Results from a workshop. }\end{array}$ & 452 \\
\hline 94 & $\begin{array}{l}\text { What proportion of patients reports long-term pain after total hip or knee replacement for osteoarthritis? A systematic review of prospective } \\
\text { studies in unselected patients. }\end{array}$ & 451 \\
\hline 95 & HOOS - validity and responsiveness in total hip replacement. & 450 \\
\hline 96 & Increased knee joint loads during walking are present in subjects with knee osteoarthritis. & 441 \\
\hline 97 & Posttraumatic osteoarthritis: a first estimate of incidence, prevalence, and burden of disease. & 441 \\
\hline 98 & Clinical practice. Osteoarthritis of the knee. & 438 \\
\hline 99 & Analysis of the discordance between radiographic changes and knee pain in osteoarthritis of the knee.J. & 438 \\
\hline 100 & The role of inflammatory and anti-inflammatory cytokines in the pathogenesis of osteoarthritis. & 438 \\
\hline
\end{tabular}

DMM, destabilization of the medial meniscus; HOOS, Hip disability, and osteoarthritis outcome score; KOOS, Knee injury and Osteoarthritis Outcome Score; OA, osteoarthritis; RCT, randomized controlled trial; WOMAC, Western Ontario and McMaster Universities Osteoarthritis Index; WORMS, Whole-Organ Magnetic Resonance Imaging Score.

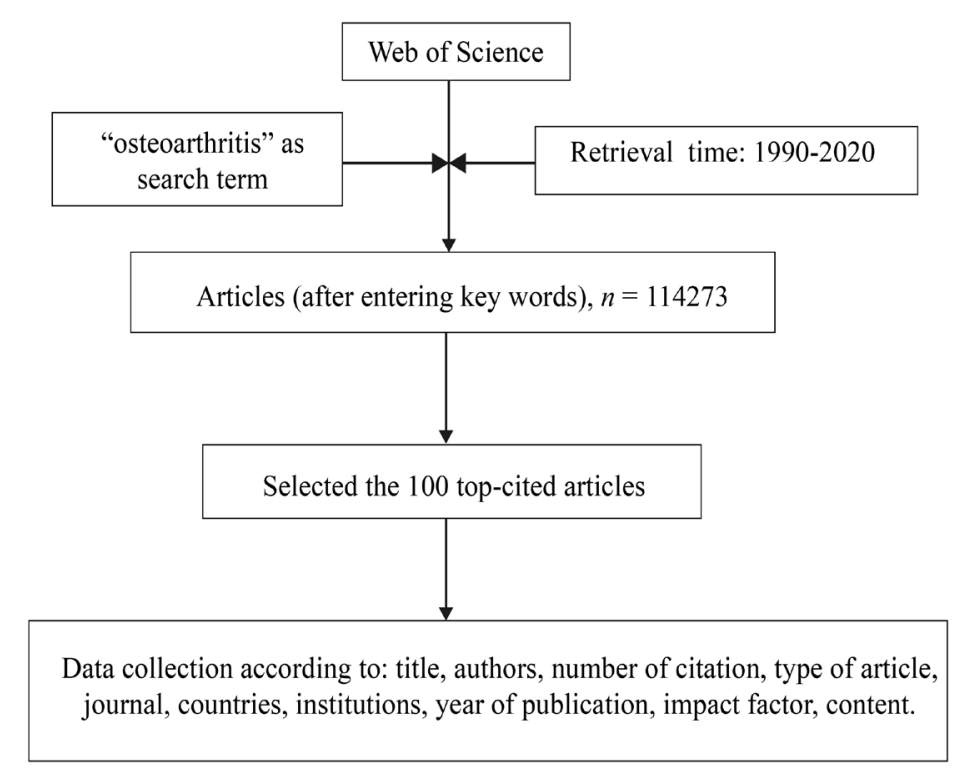

Figure 1: The process of article collection.

the full text by linking to other search platforms such as PubMed. Then we collected some information, including institutions, type of articles, countries, and study content. All the collected information was sorted into a spreadsheet and further analyzed. More details of the top 100 cited articles in the field of OA are shown in Table 1. The process of data collection is shown in Figure 1.
The types of the target articles were categorized into review, clinical research, basic science, guidelines and consensus, and other types. Clinical research was divided into a prospective study, retrospective study, randomized controlled study, and case report. The theme of these articles included etiology/harm, pathophysiology, epidemiology, diagnosis and treatment, and the prognosis of OA. 


\section{Results and Discussion}

\section{Authors, Countries and Institutions}

There were 84 first authors. And seven of them owned two or more top-cited articles. Felson DT was the first author for six articles, whose articles had the highest number of total citations. More details are shown in Table 2.

Eighteen different countries contributed to the target articles. Most of them were from The United States $(n=48)$, followed by The United Kingdom ( $n=15)$. The number of papers in each country was 48 in the United States, 15 in the United Kingdom, 8 in Canada, 7 in Sweden, 5 in France, 2 in Japan, 2 in Norway, 2 in Australia, 2 Switzerland, and 10 in others.

These articles came from 73 institutions (the institutions of the corresponding authors), led by Boston University in the United States $(n=10)$, followed by Lund University in Sweden $(n=6)$. Fourteen different institutions contributed two or more the top-cited articles, respectively. More details are shown in Table 3.

\section{Journals and Publication Years}

The 100 papers appeared in a total of 35 journals. The journal with the largest number of the target articles was Arthritis

Table 2: Authors with two or more top-cited articles

\begin{tabular}{llccc}
\hline Rank & Author & $\begin{array}{c}\text { No. of } \\
\text { articles }\end{array}$ & $\begin{array}{c}\text { Document serial } \\
\text { number }\end{array}$ & Total citations \\
\hline 1 & Felson, DT & 6 & $6,20,46,49,67,82$ & 4617 \\
2 & Altman, R & 5 & $10,32,40,51,93$ & 3819 \\
3 & Zhang, W & 4 & $4,17,56,80$ & 3687 \\
4 & Ganz, Reinhold & 2 & 2,43 & 2433 \\
5 & Goldring, Mary B & 2 & 21,59 & 1415 \\
6 & Sharma, L & 2 & 23,83 & 1321 \\
7 & Daniel, J & 2 & 63,69 & 1052 \\
\hline
\end{tabular}

\& rheumatism ( $n=20$, including before and after the name change), followed by OA and cartilage ( $n=17)$, Annals of the rheumatic disease $(n=9)$, Annals of internal medicine $(n=5)$, Lancet $(n=4)$, The New England journal of medicine $(n=4)$, The Journal of rheumatology $(n=4)$, JAMA $(n=4)$ and others $(n=32)$. The impact factors of these journals were ranging from 74.699 to 0 . More details were shown in Table 4.

The span of publication date of these target articles was from Nov 1990 to Jul 2015. We demonstrated that most of the highly cited articles $(n=74)$ were published after 2000 . Fifty-five articles were published from 2000 to 2009 and 19 articles were published in 2010 and later (Figure 2). The latest article was a clinical study on the adverse reaction of drugs in the treatment of OA, and it was published in 2015. The earliest article, which was published in 1990, was about the classification and reporting of hand OA.

Table 3: Institution with more than two articles

\begin{tabular}{llcc}
\hline Rank & Institutions & No. of articles & Total citations \\
\hline 1 & Boston University & 10 & 6816 \\
2 & Lund University & 6 & 4763 \\
3 & University of Maryland & 2 & 2221 \\
4 & University of Edinburgh & 2 & 2142 \\
5 & Bowman Gray School of Medicine & 2 & 1871 \\
6 & de l'Universite de Montreal & 2 & 1554 \\
7 & Mount Sinai Hospital & 2 & 1491 \\
8 & Keele University & 2 & 1407 \\
9 & University of lowa & 2 & 1171 \\
10 & Sorbonne Universite Univ Paris & 2 & 1146 \\
11 & Northwestern University & 2 & 1130 \\
12 & University of Southampton & 2 & 1085 \\
13 & Indiana University School of Medicine & 2 & 1084 \\
14 & University of Bristol & 2 & 1076 \\
\hline
\end{tabular}

Table 4: Journals with more than two articles

\begin{tabular}{|c|c|c|c|c|}
\hline Rank & Journals & 2019 Impact Factor & No. of articles & The serial number of articles \\
\hline 1 & Arthritis and rheumatism & 9.586 & 20 & $1,2,7,8,9,17,19,21,22,23,28,30,31,35,39,40,50,54,55,91$ \\
\hline 2 & Osteoarthritis and cartilage & 4.793 & 17 & $6,12,44,46,53,59,65,66,74,75,76,77,81,83,84,94,96$ \\
\hline 3 & Annals of the rheumatic disease & 16.102 & 9 & $36,42,48,58,62,63,64,95,97$ \\
\hline 4 & Annals of internal medicine & 21.317 & 5 & $15,27,37,51,99$ \\
\hline $5 a$ & Lancet & 60.392 & 4 & $33,61,88,100$ \\
\hline $5 b$ & The New England journal of medicine & 74.699 & 4 & $3,41,67,71$ \\
\hline $5 c$ & The Journal of rheumatology & 3.35 & 4 & $4,16,32,69$ \\
\hline $5 d$ & JAMA-journal of the American medical association & 45.54 & 4 & $13,26,29,34$ \\
\hline 6 & The Journal of Clinical Investigation & 11.864 & 3 & $5,10,14$ \\
\hline $7 a$ & Clinical orthopedics and related research & 4.329 & 2 & 47,78 \\
\hline $7 b$ & American journal of sports medicine & 5.81 & 2 & 72,80 \\
\hline 7c & Nature reviews rheumatology & 16.625 & 2 & 85,87 \\
\hline $7 d$ & The journal of bone and joint surgery & 0 & 2 & 24,57 \\
\hline
\end{tabular}




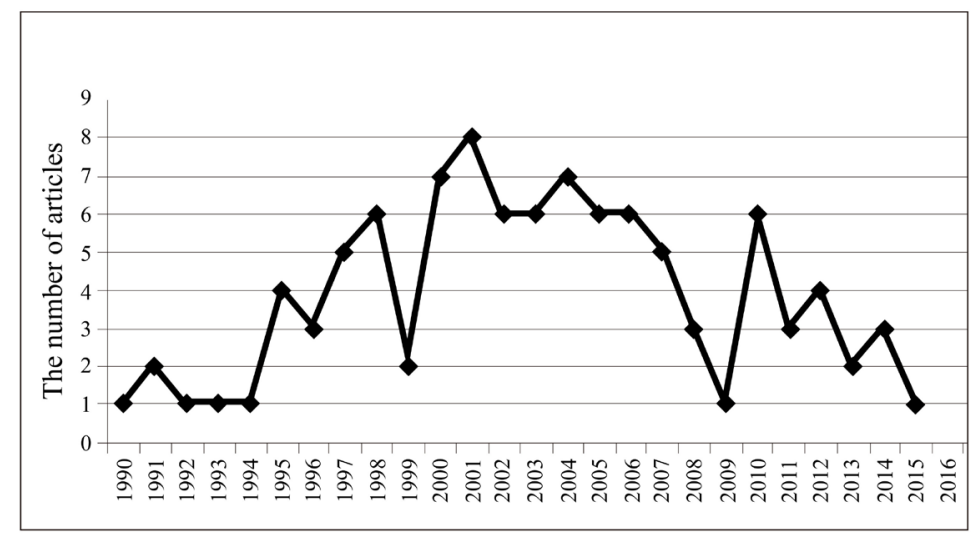

Figure 2: The number of articles in different years.

A

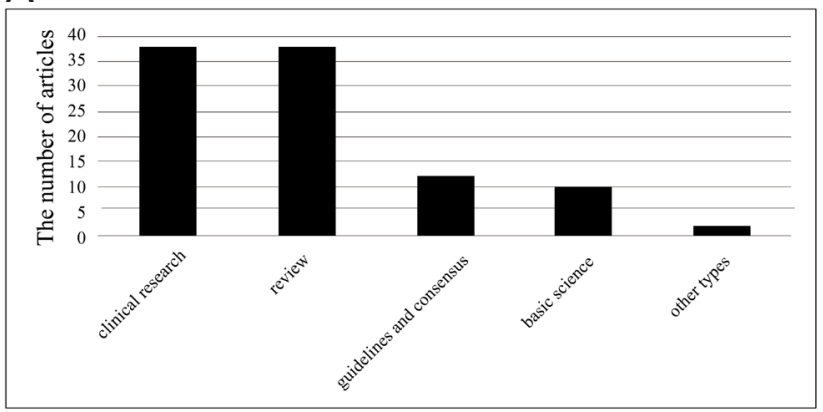

B

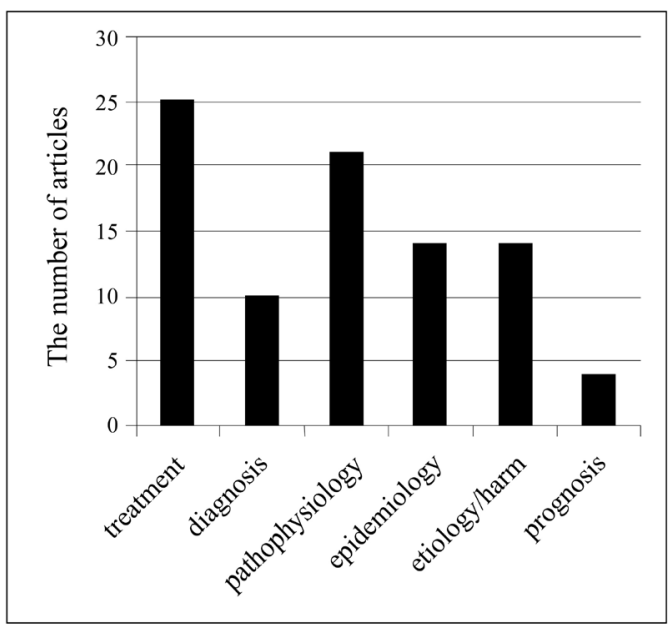

Figure 3: (A) The type of articles. (B) The content of articles.

\section{Type of Article and Theme of Study}

The 100 top-cited articles covered several types of articles, including clinical research $(n=38)$, review $(n=38)$, guidelines and consensus $(n=12)$, basic science $(n=10)$ and other types $(n=2)$ (Figure 3A). Clinical studies were divided into prospective study $(n=19)$, retrospective study $(n=11)$, randomized controlled trial (RCT) $(n=7)$ and case report $(n=1)$. The theme of these studies on OA mainly focused on treatment $(n=25)$, followed by pathophysiology $(n=21)$, etiology/harm $(n=14)$, epidemiology $(n=14)$, diagnosis $(n=10)$, prognosis $(n=4)$ and others (Figure 3B).

\section{Discussion}

$\mathrm{OA}$ was an age-related disease, and it was the highest incidence rate of osteoarthropathy worldwide, especially the $O A$ of hip and knee joints, which were the main cause of global disability. It had a significant impact on individual well-being and increased the social and individual economic burden. Therefore, more researches were needed to guide the prevention, diagnosis, and treatment of OA. The purpose of this study was to analyze the 100 top-cited articles in the field of $\mathrm{OA}$ at home and abroad in the past 30 years, hoping to provide a valuable reference for further research on OA.

Although citations are not the only criterion for judging the importance of an article, it was usually used to identify very useful papers and regarded as a proxy to the academic value and influence of an article to a certain extent. The highest cited article, authored by Silverstin, was cited 2382 times. The reasons why it was quoted most frequently might be: (1) Its content was a hot topic in clinical practice; (2) Its type of study was a randomized controlled trial with the highest quality of evidence; (3) It was published in a journal with high impact factor; (4) It was published for 20 years, and has enough time to accumulate citations. Many factors were affecting the number of citations of articles, one of which was the impact factor of the published journals. The higher the impact factor of a journal, the more likely its articles would be concerned and cited. It was worth mentioning that Arthritis \& 
Rheumatism (renamed Arthritis \& Rheumatology) contributed to most articles in this study. Although its impact factor was not the highest, it was the official journal of the American College of Rheumatology, and its impact factor ranked the top three in similar journals in rheumatism. Therefore, many target articles were published in this journal.

The main types of articles were clinical studies $(n=38)$ and reviews $(n=38)$. Unsurprisingly, the institution with the largest number of articles was Boston University in the United States. The United States had the largest number of cited articles in the field of OA, as well as in most medical fields.

The span of the publication date of these target articles was from Nov 1990 to Jul 2015. Most of the highly-cited articles ( $n=74$ ) were published after 2000. Taking a five-year time frame, we found that the five-year period from 2000 to 2004 saw the largest number of these target articles $(n=34$, total impact factor $=528.696)$, followed by $2005-2009$ $(n=21$, total impact factor $=369.643), 1995$ to $1999(n=20$, total impact factor $=177.638), 2010$ to 2014 ( $n=18$, total impact factor $=186.081), 1990-1994(n=6$, total impact factor $=130.402)$ and after $2015(n=1$, total impact factor $=60.392$ ). It was not difficult to see that the total impact factor in different periods was closely related to the number of articles and the impact factor of each journal.

The theme of these studies on OA mainly focused on treatment $(n=25)$ and pathophysiology $(n=21)$. Secreted inflammatory molecules were among the critical mediators of the disturbed processes implicated in OA pathophysiology. Interleukin (IL)-1 beta and tumor necrosis factor (TNF), in particular, control the degeneration of the articular cartilage matrix. ${ }^{[12]}$ Treatment options included non-drug therapy, drug therapy, and surgical therapy. The treatments discussed in this article included cartilage protectant $(n=5)$, weight loss and exercise $(n=3)$, joint replacement $(n=2)$, and comprehensive therapy $(n=15)$. The treatment of knee and hip joint were mainly discussed. Joints nutritional supplements could be used in mild to moderate doses for patients to relieve pain and improve function. ${ }^{[13]}$ Cartilage protectant was mainly discussed with glucosamine sulfate. For people with body mass index $\geq 28 \mathrm{~kg} / \mathrm{m}^{2}$, researches have shown that combining modest weight loss provided the best overall improvements in self-reported measures of function and pain and performance measures of mobility. ${ }^{[14]}$ For those patients with hip and knee OA who were not obtaining adequate pain relief and functional improvement with a combination of pharmacological and non-pharmacological treatments, there were five main surgical modalities: total joint replacements, unicompartmental knee replacement, osteotomy, and joint preserving surgical procedures; joint lavage and arthroscopic debridement in knee OA, and joint fusion as a salvage procedure when joint replacement had failed. ${ }^{[15]}$ It was worth noting that the treatment should be tailored to the specific needs of different patients. Many factors need to be taken into account, such as risk factors, location of structural damage, pain intensity, degree of dysfunction, patient's expectations, and so on.

It should be kept in mind that this study had some limitations. First, this study only searched articles on the web of science, neglecting the articles published in the printed version, which might omit some important articles. Second, the number of citations was sometimes different according to the citation database, such as the Web of Science and Google Scholar. Third, they were limited to English, and some important articles published in other languages might be omitted. Furthermore, there were also other potential elements. For example, time might affect the number of citations. The recent articles might have inadequate time to accumulate the number of citations, although total citation times were not obviously associated with the age of the article. ${ }^{[16]}$ Despite limitations, we believed this study could contribute to obtaining vital developments of OA and providing new insights into innovation in this field.

\section{Conclusion}

This study identified the top 100 most cited articles in OA in recent 30 years and analyzed their bibliometric characteristics, which may pave the way for further research. Most of the articles were published after 2000. But the latest articles may not be included in the top 100 cited articles, because recent studies did not have enough time to accumulate the number of citations.

\section{Acknowledgment}

The authors acknowledge and thank all members who helped complete this article.

Conflict of Interest

The authors have declared no conflicts of interest.

Funding or Financial Support

This work was supported by the Project of Youth Innovation in Medical Research in Sichuan Province (Q15027), the Project of Sichuan Edu- 
cation Department (18ZB0640), the Project of Health Department in Sichuan Province (150078), Doctoral Foundation of Affiliated Hospital of Southwest Medical University (No. 18048), the Project of Technology Department in Sichuan Province(20YYJC2015) and the Project of Southwest Medical University (07092).

Author Contributions

Feng Yin and Qiuyu Yang wrote the manuscript; Chengsong He and Jie Chen revised the manuscript; All authors read and approved the final manuscript. Conceptualization: Feng Yin. Data curation: Feng Yin, Qiuyu Yang, Yue He, Lihui Peng, Zixia Zhao. Formal analysis: Feng Yin, Jie Chen. Funding acquisition: Jie Chen. Investigation: Feng Yin, Jie Chen. Methodology: Feng Yin, Qiuyu Yang, Yue He, Lihui Peng, Zixia Zhao, Chengsong He, Jie Chen. Resources: Jie Chen. Writing - original draft: Feng Yin, Qiuyu Yang, Jie Chen. Writing - review \& editing: Feng Yin, Qiuyu Yang, Yue He, Lihui Peng, Zixia Zhao, Chengsong He, Jie Chen.

\section{References}

[1] Grandi FC, Bhutani N. Epigenetic Therapies for Osteoarthritis. Trends Pharmacol Sci. 2020;41:557-569.

[2] David RV, Cristina GM, Sonia PD, et al. Prevalence of Osteoarthritis of the Knee in A Random Population Sample in People Aged 40 Years and Over. Gac Med Mex. 2019;155:39-45.

[3] Tarride JE, Mahbubul Haq, O'Reilly DJ, et al. The Excess Burden of Osteoarthritis in the Province of Ontario, Canada. Arthritis Rheumatol. 2012;64:1153-1161.

[4] Busija L, Buchbinder R, Osborne RH. A Grounded Patient-Centered Approach Generated the Personal and Societal Burden of Osteoarthritis Model. J Clin Epidemiol. 2013;66:994-1005.

[5] Ahmad I, Ahmed G, Shah SAA, et al. A Decade of Big Data Literature: Analysis of Trends in Light of Bibliometrics. J Supercomput. 2020;76:3555-3571.

[6] Hou D, Bi X, Mao Z, et al. Biomaterials Research of China from 2013 to 2017 Based on Bibliometrics and Visualization Analysis. Peer J. 2019;7:e6859.

[7] He LG, Fang H, Wang XL, et al. The 100 Most-Cited Articles in Urological Surgery: A Bibliometric Analysis. Int J Surg. 2020;75:74-79.

[8] Ahmad P, Dummer PMH, Chaudhry A, et al. A Bibliometric Study of the Top 100 most-cited Randomized Controlled Trials, Systematic Reviews and Meta-Analyses Published in Endodontic Journals. Int Endod J. 2019;52:1297-1316.
[9] Yuan FF, Cai JZ, Liu B, Tang XW. Bibliometric Analysis of 100 Top-Cited Articles in Gastric Disease. Biomed Res Int. 2020;2020:2672373.

[10] Park BS, Kim IH, Park KM. Top 100 Cited Articles on Sleep Medicine: A Bibliometric Analysis. Eur Neurol. 2020;83:111-119.

[11] Connelly TM, Malik Z, Sehgal R, Byrnes G, et al. The 100 most Influential Manuscripts in Robotic Surgery: A Bibliometric Analysis. J Robot Surg. 2020;14:155-165.

[12] Mohit K, Johanne MP, Daniel L, et al. Role of Proinflammatory Cytokines in the Pathophysiology of Osteoarthritis. Nat Rev Rheumatol. 2011;7:33-42.

[13] Reginster JY, Deroisy R, RovatiL C, et al. Long-Term Effects of Glucosamine Sulphate on Osteoarthritis Progression: A Randomised, Placebo-Controlled Clinical Trial. Lancet. 200127;357:251-256.

[14] Stephen PM, Richard FL, Gary DM, et al. Exercise and Dietary Weight Loss in Overweight and Obese Older Adults with Knee Osteoarthritis. Arthritis Rheum. 2004;50:1501-1510.

[15] Zhang W, Moskowitz RW, Nuki G, et al. OARSI Recommendations for the Management of Hip and Knee Osteoarthritis, Part II: OARSI Evidence-Based, Expert Consensus Guidelines. Osteoarthritis Cartilage. 2008;16(2):137-162.

[16] Yu J, Hu R, Zhu GX. Top 100 Cited Articles on Infection in Orthopaedics A Bibliometric Analysis. Medicine. 2019;98:e14067. 